\title{
Application of Electrical Effective Channel Length and External Resistance Measurement Techniques to a Submicrometer CMOS Process
}

\author{
DAVID J. MOUNTAIN
}

\begin{abstract}
Several new methods to extract the effective channel length ( $\left.L_{\text {eff }}\right)$ and series resistance $\left(R_{\text {ext }}\right)$ parameters for MOS devices have been proposed recently. This is a result of inaccuracies in standard techniques as device lengths are reduced to $1.0 \mu \mathrm{m}$ and lower, especially with the introduction of lightly doped drain (LDD) transistor structures. In this study, several methods are applied to MOSFET's fabricated in a submicrometer CMOS process, and the limitations of the extraction routines are analyzed and compared. It is found that the limiting factors are the applicability of the $V_{t}$ calculations and the precision of the $I_{d s}$ measurements.
\end{abstract}

\section{INTRODUCTION}

$I^{\prime}$ $\mathrm{N}$ the past few years, several techniques [1]-[3] have been presented that measure the effective channel length $\left(L_{\text {eff }}\right)$ and external resistance ( $\left.R_{\text {ext }}\right)$ of MOS transistors. They have been developed because of the inaccuracy involved in using standard methods as MOSFET channel dimensions are reduced to below $1.0 \mu \mathrm{m}$. Also, the use of lightly doped drain (LDD) structures has made measurement of the parasitic resistance more important for modeling transistor performance [4], [5]. An analysis of several electrical techniques using device simulation indicated that various physical factors influenced parameter extraction routines and suggested some modifications to correct these problems [6], [7]. In this study, various techniques are used to measure $L_{\text {eff }}$ and $R_{\text {ext }}$ for both conventional and LDD MOSFET's fabricated in a submicrometer CMOS process, and the limitations involved in their application are discussed.

\section{Extraction Methods}

For an MOS device operating in the linear region, the total dc resistance can be modeled as [8]

$$
R_{d s}=V_{d s} / I_{d s}=A \times\left(L_{\mathrm{eff}}\right)+R_{\mathrm{ext}}
$$

since

$$
L_{\text {eff }}=L_{m}-d L
$$

we can write

$$
R_{d s}=A \times L_{m}+B
$$

Manuscript received February 1, 1989; revised June 5, 1989. The review of this paper was arranged by Associate Editor R. R. Troutman.

The author is with the Department of Defense, Fort Meade, MD 20755 IEEE Log Number 8930575. where

$$
B=-A \times d L+R_{\mathrm{ext}}
$$

and

$$
A=\left[\mu_{\mathrm{eff}} \times C_{\mathrm{ox}} \times W_{\mathrm{eff}} \times\left(V_{g s}-V_{t}-0.5 \times V_{d s}\right)\right]^{-1} .
$$

$L_{m}$ is the mask length of the device and $d L$ is the reduction of the length from $L_{m}$ due to poly undercut, source/drain diffusion, etc. All other symbols have their usual meanings. A plot of $R_{d s}$ versus $L_{m}$ can be fitted by standard linear regression algorithms [9] to find both the $A$ (slope) and $B$ ( $y$-intercept) parameters. If a series of differing bias conditions is applied, a family of $R_{d s}-L_{m}$ lines can be used to obtain a set of $(A, B)$ points. Analyzing a plot of $B$ versus $A$ by regression can determine the values of $d L$ (slope) and $R_{\text {ext }}(y$-intercept). A more complete treatment of the extraction method and its assumptions can be found in [8]. The most questionable assumption in the model is that $d L$ and $R_{\mathrm{ext}}$ are constants for the range of biases used. It has been shown that this may not be the case, especially for LDD devices [5], [6]. For this reason, the use of small bias ranges for individual $d L$ and $R_{\text {ext }}$ calculations is preferable.

There are essentially two ways to vary the bias conditions to create this family of $R_{d s}$ versus $L_{m}$ lines:

1) fix $V_{b s}$ and vary $V_{g s}$, as in [1] or

2) fix $V_{g s}$ and vary $V_{b s}$, as in [4].

It was noted [1], [8] that if the devices used had differing $V_{t}$ values, the proper method was to adjust $V_{g s}$ so that $V_{g s}$ $-V_{t}=V_{g s t}$ was equal for all transistor lengths. This new method, termed the gate-drive (GD) technique, then varies $V_{g s t}$ to produce the data needed for parameter extraction. Fig. 1 shows the use of the GD method to obtain $d L$ and $R_{\text {ext }}$ for a conventional MOS device. In order to eliminate the geometry (or short-channel) effect associated with substrate bias methods, a substrate-bias gate-drive (SBGD) routine was developed [6], [7]. In this method, $V_{g s}$ of the longest device remains constant for all substrate biases. Since $V_{t}$ varies with $V_{b s}$, each set of $R_{d s}-L_{m}$ data in the SBGD method is found from a particular $\left(V_{g s t}, V_{b s}\right)$ combination. Fig. 2(a) and (b) displays the application of the SBGD method. 


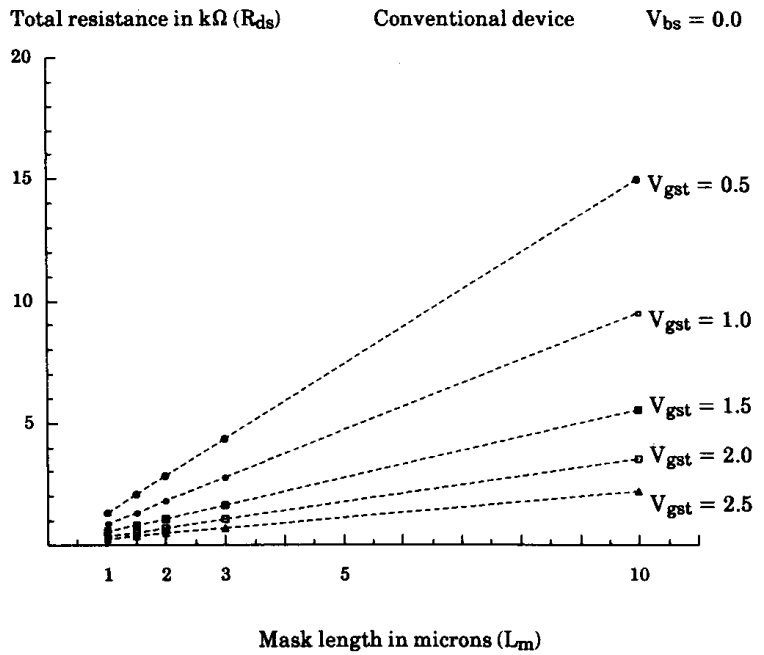

(a)

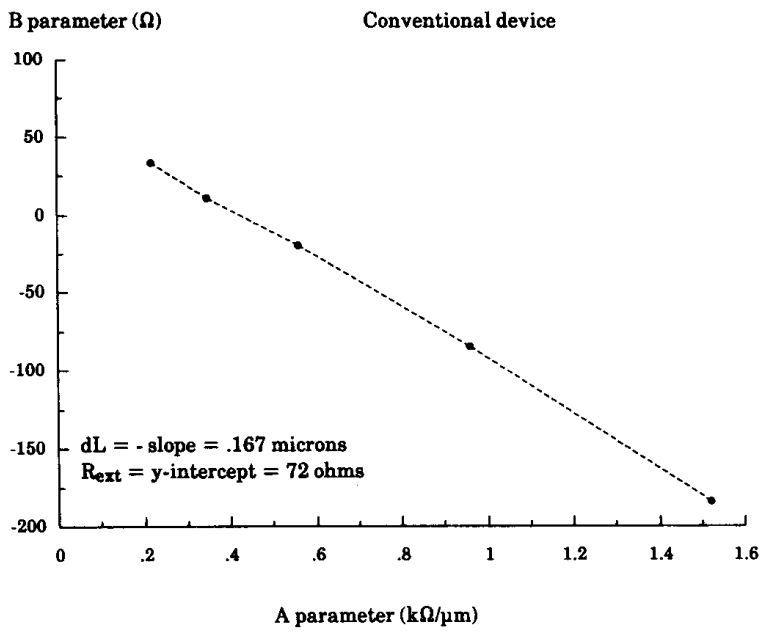

(b)

Fig. 1. Gate-drive (GD) method applied to conventional MOS device (a) to obtain $A$ and $B$ parameters and (b) to obtain $d L, R_{\text {ext }}$ values.

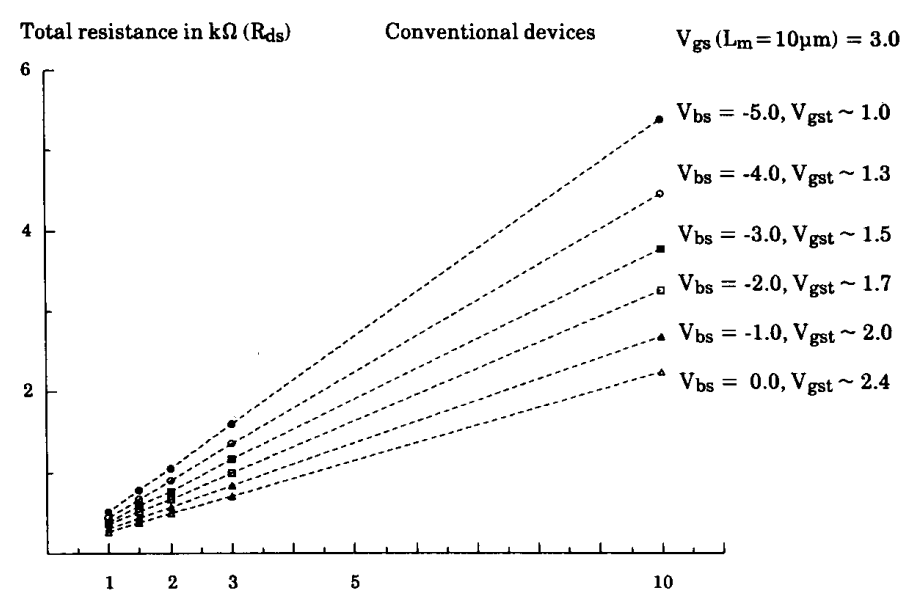

Mask length in microns $\left(\mathrm{L}_{\mathrm{m}}\right)$

(a)

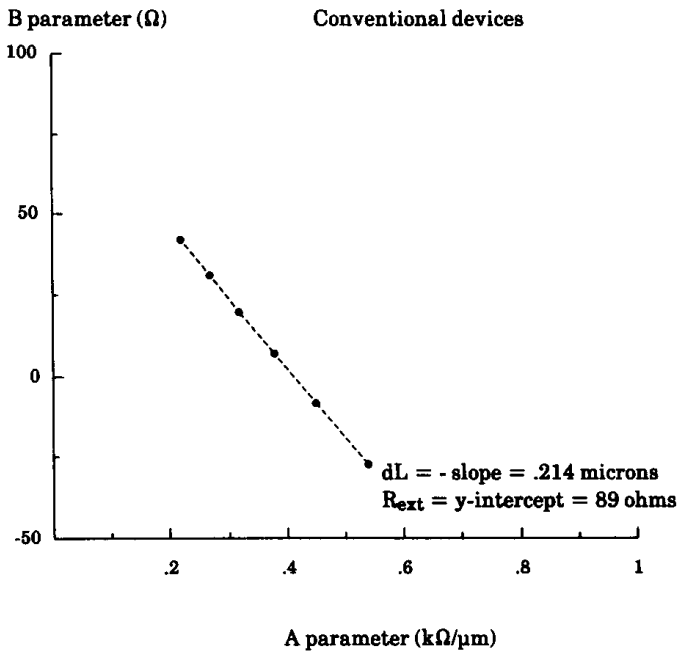

(b)

Fig. 2. Substrate-bias gate-drive (SBGD) method applied to (a) obtain $A$ and $B$ parameters and (b) to obtain $d L, R_{\text {ext }}$ values.

The determination of $V_{t}$ for each transistor was also found to be critical, especially when using small gate drives [7]. A standard technique for obtaining $V_{t}$ uses the linear region $I_{d s}-V_{g s}$ curve. At the point of maximum transconductance $\left(G_{m}=\Delta I_{d s} / \Delta V_{g s}\right)$, the curve is linearly extrapolated to zero current. The gate voltage corresponding to this point is the threshold voltage. This method was shown to be sensitive to $R_{\text {ext }}$, although it was noted that a first-order resistance correction adjusted $V_{t}$ to give satisfactory results [7]. The authors suggested that the use of a fixed-current method, where $V_{t}$ is the gate voltage corresponding to a specified $I_{d s}$ (normalized to unit $W_{m} / L_{m}$ ), was more consistent.

\section{Experimental Detalls}

Both conventional and LDD devices with $L_{m}=1.0$, $1.5,2.0,3.0$, and $10.0 \mu \mathrm{m}$, and $W_{m}=25.0 \mu \mathrm{m}$ were used for the $L_{\text {eff }}$ and $R_{\text {ext }}$ measurements. These devices were built in a submicrometer CMOS process. Table I shows some processing details for the three NMOS device structures (CONV, LDD1, LDD2) fabricated. The p-channel transistors did not have LDD structures. Table II shows parametric data for $L_{m}=0.6 \mu \mathrm{m}$ devices made in this process. The linear region data for the techniques was taken using an HP4145 Semiconductor Parameter Analyzer controlled by an HP1000 computer. The biases applied (with opposite polarity for PMOS) were $V_{b s}=0$ to 


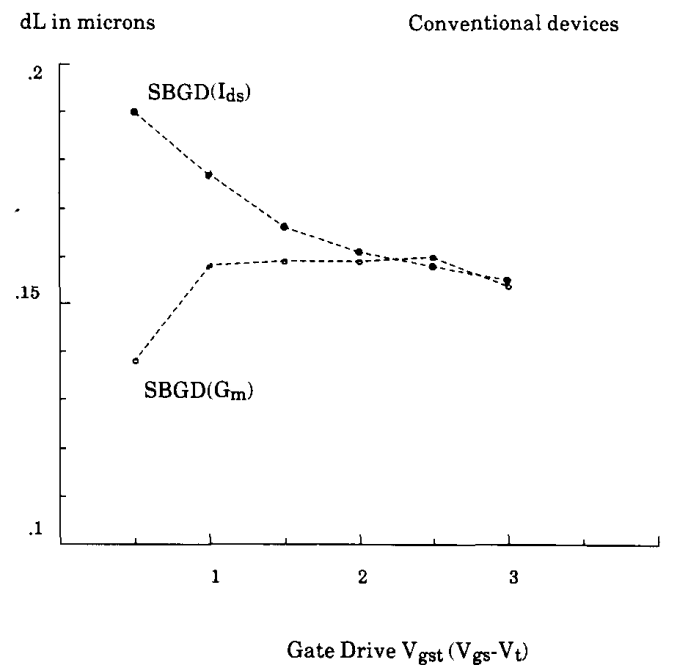

(a)

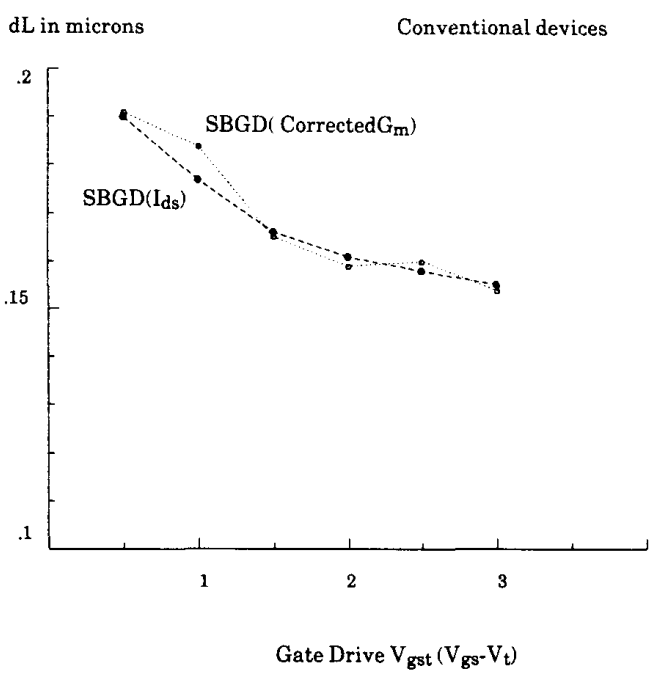

(b)

Fig. 3. (a) Comparison of different $V$, techniques using SBGD method and conventional MOS devices. (b) Use of the corrected $G_{m}$ technique for the SBGD method and conventional MOS devices.

TABLE I

Processing Information for the Three Device Types Used in this STUDY

\begin{tabular}{l|c|c|c}
\hline \hline & CONV & LDD1 & LDD2 \\
\hline$T_{\text {ox }}(\mathrm{nm})$ & 19 & 19 & 19 \\
\hline Spacer $(\mu \mathrm{m})$ & None & 0.1 & 0.15 \\
\hline $\mathrm{N} \cdot$ dose $\left(\# / \mathrm{cm}^{2}\right)$ & None & $1 \times 10^{13}$ & $1 \times 10^{13}$ \\
\hline $\mathrm{N} \cdot$ energy $(\mathrm{keV})$ & None & 30 & 45 \\
\hline $\mathrm{P} \cdot$ dose $\left(\# / \mathrm{cm}^{2}\right)$ & None & None & None \\
\hline
\end{tabular}

TABLE II

n-Channel and p-Channel Parametric Data for all Three Mos STRUCTURES FOR $L_{m}=0.6 \mu \mathrm{m}$

\begin{tabular}{l|c|c|c|c|c|c}
\hline & \multicolumn{2}{|c|}{ CONV } & \multicolumn{2}{c|}{ LDD1 } & \multicolumn{2}{c}{ LDD2 } \\
\cline { 2 - 8 } & NMOS & PMOS & NMOS & PMOS & NMOS & PMOS \\
\hline $\mathrm{I}_{\text {dsat }}(\mathrm{mA})$ & 12.0 & 7.6 & 9.1 & 5.6 & 9.0 & 4.8 \\
\hline $\mathrm{V}_{\mathrm{t}}($ volts $)$ & .442 & -.63 & .548 & -922 & .494 & .943 \\
\hline $\mathrm{G}_{\mathrm{m}}(\mu \mathrm{A} / \mathrm{V})$ & 198 & 82 & 171 & 69 & 172 & 62 \\
\hline SubVt slope (mV/decade) & 97 & 104 & 94 & 122 & 94 & 112 \\
\hline
\end{tabular}

$-5 \mathrm{~V} ; V_{g s}$ was 0 to $5 \mathrm{~V}$ in $50-\mathrm{mV}$ increments; $V_{d s}=50$ $\mathrm{mV}$ for all data points. The GD and SBGD methods were analyzed using both fixed-current $\left(I_{d s}=0.2 \mu \mathrm{A} \times\right.$ $\left.W_{m} / L_{m}\right)$ and maximum $G_{m}$ techniques for determination of the threshold voltage. This resulted in four methods being compared:

1) GD using fixed-current $V_{t}: \quad \operatorname{GD}\left(I_{d s}\right)$

2) SBGD using fixed-current $V_{t}: \quad \operatorname{SBGD}\left(I_{d s}\right)$

3) GD using maximum slope $V_{t}: \quad \operatorname{GD}\left(G_{m}\right)$

4) SBGD using maximum slope $V_{i}: \operatorname{SBGD}\left(G_{m}\right)$
Approximately ten sites per device type were tested to obtain the $d L, R_{\text {ext }}$ parameters. The values shown in the figures and tables are the averages for all sites. It has been noted [8] that the selection of device lengths and applied biases can affect the results of the regression analyses. While small variations in $d L$ and $R_{\text {ext }}$ occurred if the selection of mask lengths and bias conditions was changed, the overall trends were not altered.

\section{Results and ANalysis}

Fig. 3(a) shows the $d L$ versus $V_{g s t}$ measurements for the CONV MOSFET's using the $\operatorname{SBGD}\left(I_{d s}\right)$ and $\operatorname{SBGD}\left(G_{m}\right)$ techniques. For $V_{g s t}>1 \mathrm{~V}$, the agreement is very good, but for smaller $V_{g s t}$, the $\operatorname{SBGD}\left(I_{d s}\right)$ method indicates a slight increase in $d L$ while $\operatorname{SBGD}\left(G_{m}\right)$ shows a small decrease. Fig. 4(a), a plot of $R_{\text {ext }}$ versus $V_{g s t}$ for LDD1 transistors shows that the $\operatorname{GD}\left(I_{d s}\right)$ and $\operatorname{GD}\left(G_{m}\right)$ techniques follow the same pattern. In fact, this pattern emerged for both $d L$ and $R_{\text {ext }}$ for all three device types. The modulation of source/drain carrier concentrations by gate bias, which occurs in an LDD transistor [5], would cause $d L$ and $R_{\text {ext }}$ to increase for smaller $V_{g s t}$. This suggests that the $\operatorname{SBGD}\left(G_{m}\right)$ and $\operatorname{GD}\left(G_{m}\right)$ methods predict incorrect trends at the lower $V_{g s t}$ values. The cause of this inconsistency is the $V$ calculation since it is the only difference between these methods and the others used. It is not surprising that the fixed-current methods are more accurate at small $V_{g s t}$ values when you consider how the different $V_{t}$ calculations fit the linear region model. The $d L, R_{\text {ext }}$ extraction methods used assume that the intrinsic channel resistance/ unit channel length ( $A$ parameter) is equal for all devices at a given bias $\left(V_{g s t}, V_{b s}\right)$ condition. Using a fixed-current $V_{t}$ calculation requires the total resistance/unit mask length to be equal for all devices at $V_{g s}=V_{t}$, while the $G_{m}$ ex- 


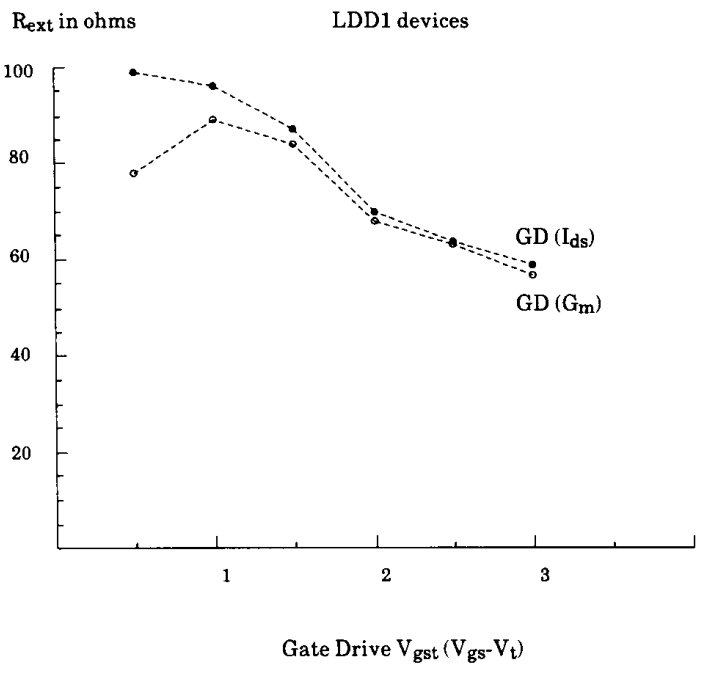

(a)

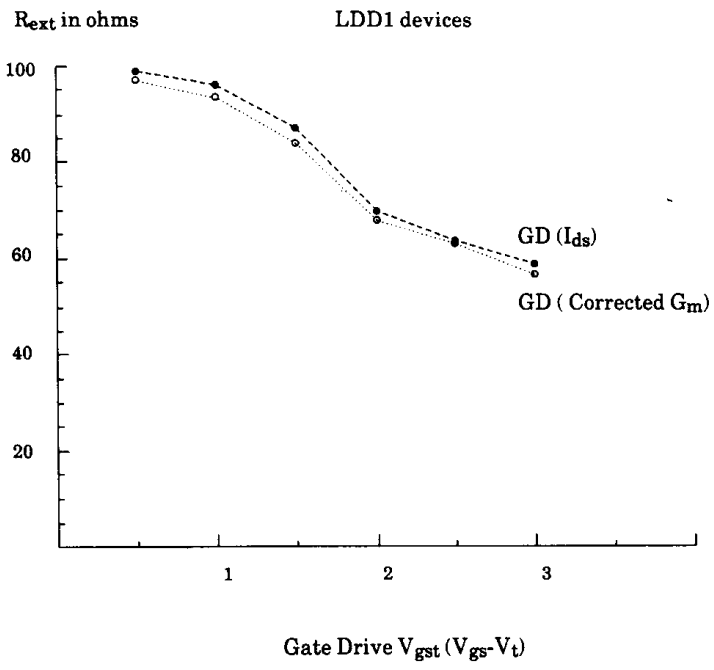

(b)

Fig. 4. (a) Comparison of different $V_{t}$ techniques using GD method and LDD1 devices. (b) Use of the corrected $G_{m}$ technique for the GD method and LDDI devices.

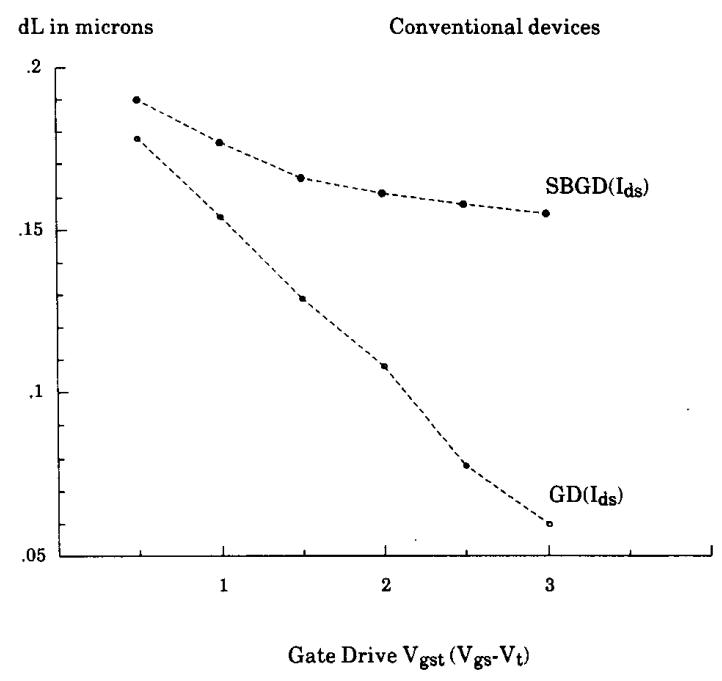

Fig. 5. Comparison of $\operatorname{SBGD}\left(I_{d s}\right)$ and $\operatorname{GD}\left(I_{d s}\right)$ methods using conventional MOS devices.

trapolation only assumes this to be true at the point of maximum transconductance. The inclusion of biases near this gate voltage (typically $V_{g s} \sim V_{t}+0.5 \mathrm{~V}$ ) requires using $I_{d s}$ points that do not have equivalent intrinsic resistance/unit channel length values, and therefore the regression results are incorrect. Correcting $G_{m}$ for the series resistance removes this source of error. Using the corrected $V_{t}$ calculation proposed in [7], we see (Figs. 3(b), 4(b) that equivalent $d L$ and $R_{\text {ext }}$ values will be found using either the fixed-current or corrected $G_{m}$ calculations of $V_{t}$. For $V_{g s t}<0.5 \mathrm{~V}$, the fixed-current and corrected $G_{m}$ methods also project nonphysical behavior, which implies

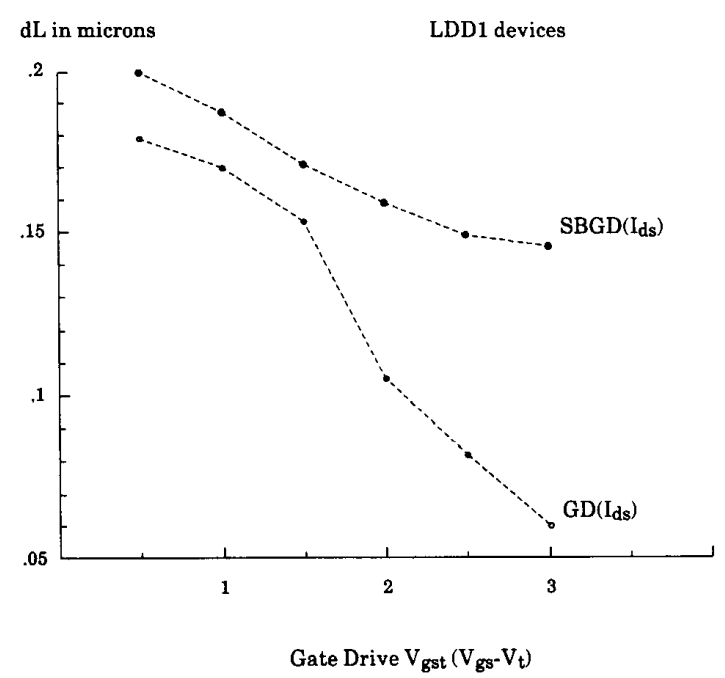

Fig. 6. Comparison of $\operatorname{SBGD}\left(I_{d s}\right)$ and $\operatorname{GD}\left(I_{d s}\right)$ methods using LDD1 devices.

that the techniques violate the model assumptions at very low gate drives.

Figs. 5 and 6 plot $d L$ versus $V_{g s t}$ data using the $\operatorname{SBGD}\left(I_{d s}\right)$ and $\operatorname{GD}\left(I_{d s}\right)$ methods for the CONV and LDD1 devices. On both plots, the GD technique indicates a larger change of $d L$ and also depicts a sharp drop for $V_{g s t}>2 \mathrm{~V}$. Fig 7 shows $R_{\text {ext }}$ versus $V_{g s t}$ values for these two techniques using LDD2 data. Here the curves are in good agreement with each other although the $\mathrm{GD}\left(I_{d s}\right)$ data is not as smooth. Fig. 8 is a plot of the $B$ versus $A$ linear correlation coefficient [9] versus $V_{g s t}$ for the two methods and all three device structures. For $V_{g s t}>2.0 \mathrm{~V}$, the cor- 


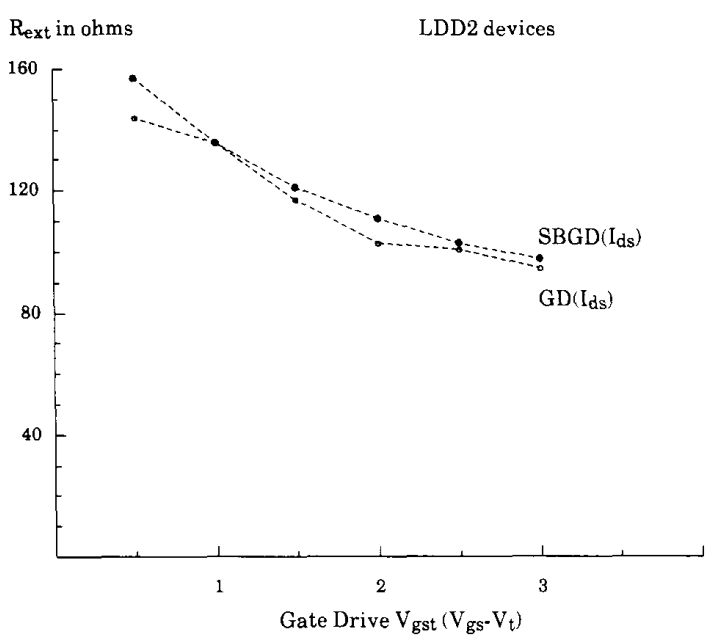

Fig. 7. Comparison of $\operatorname{SBGD}\left(I_{d s}\right)$ and $\operatorname{GD}\left(I_{d s}\right)$ methods using LDD2 devices.

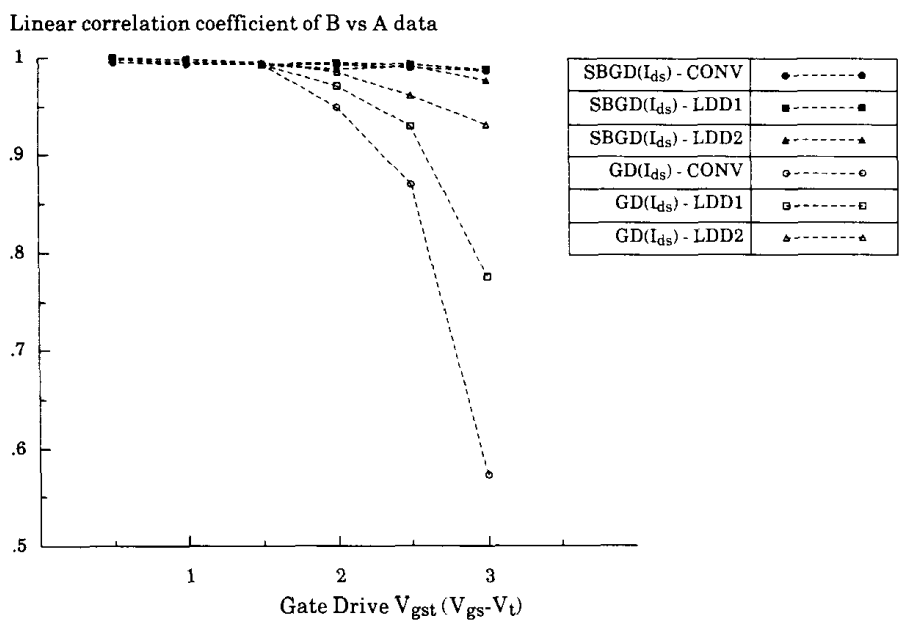

Fig. 8. Correlation of second regression versus $V_{g s t}$ for all device types using SBGD and GD methods.

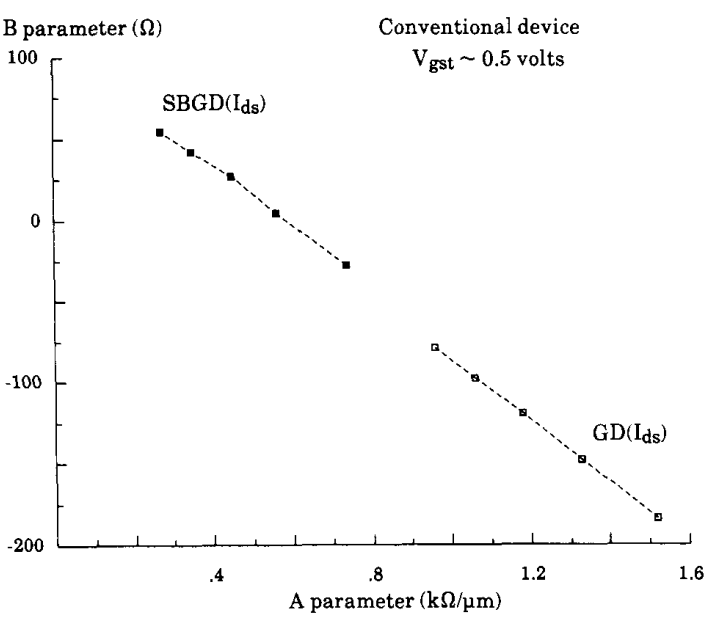

Fig. 9. Plot of $B$ versus $A$ parameters for $V_{g s t} \sim 0.5 \mathrm{~V}$ and conventional MOS devices. relation coefficient for the GD $\left(I_{d s}\right)$ method starts to decrease rapidly. Figs. 9 and 10 explain why. For $V_{g s t} \simeq$ $0.5 \mathrm{~V}$, the range of $(A, B)$ values is very large (Fig. 9), but it is much smaller at $V_{g s t} \simeq 2.5 \mathrm{~V}$ (Fig. 10), especially for the GD $\left(I_{d s}\right)$ method. Deviations in $R_{d s}$ measurements that cause slight inaccuracy in the calculated values for $A$ and $B$ can have a substantial impact on the slope $(d L)$ at high $V_{g s t}$. These inaccuracies cause the data to be less linear, resulting in a lower correlation coefficient. However, the extrapolation of this inaccurate slope to find the $y$-intercept $\left(R_{\text {ext }}\right)$ is not large enough to significantly alter the $R_{\text {ext }}$ value. Since the $\operatorname{SBGD}\left(I_{d s}\right)$ technique fits the $B$ versus $A$ regression over a slightly wider range of values at high gate drives, it is less affected by this influence. However, a limit of $V_{g s t} \sim 3.5 \mathrm{~V}$ appears to exist for $\operatorname{SBGD}\left(I_{d s}\right)$ methods.

The four methods were also applied to PMOS transistors fabricated in these processes. The trends at high and 


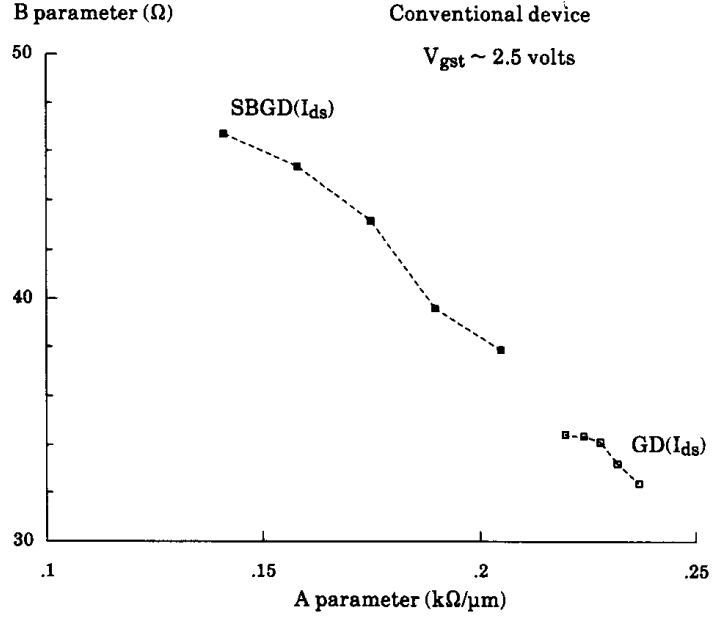

Fig. 10. Plot of $B$ versus $A$ parameters for $V_{g s t} \sim 2.5 \mathrm{~V}$ and conventional MOS devices.

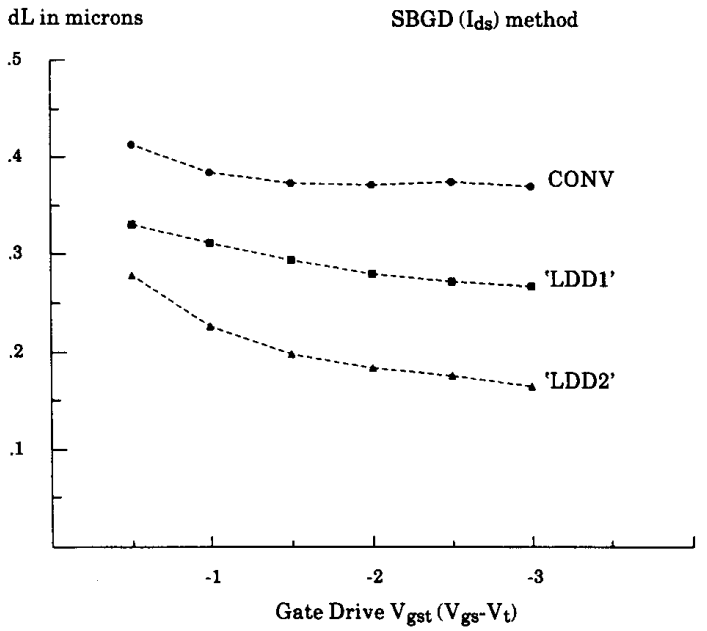

Fig. 11. Use of the $\operatorname{SBGD}\left(I_{d s}\right)$ method to find $d L$ for p-channel devices.

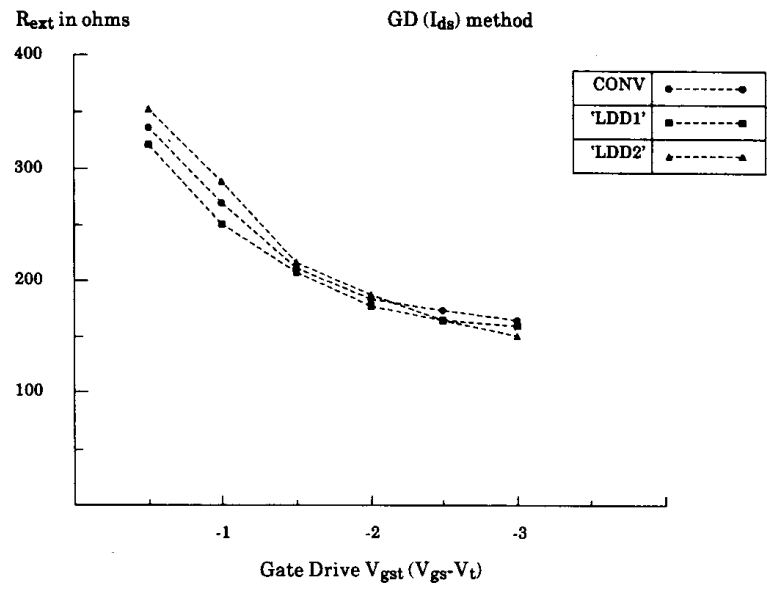

Fig. 12. Use of the $\mathrm{GD}\left(I_{d s}\right)$ method to find $R_{\text {ext }}$ for p-channel devices.
TABLE III

VAlues for $d L(\mu \mathrm{m})$ versus $V_{\text {gst }}$ For All Three Processes Using the $\operatorname{SBGD}\left(I_{d s}\right)$ TECHNIQUE

\begin{tabular}{c|c|c|c|c|c|c}
\hline \hline \multirow{2}{*}{$\left|V_{\text {gst }}\right|$} & \multicolumn{2}{|c|}{ CONV } & \multicolumn{2}{c|}{ LDD1 } & \multicolumn{2}{c}{ LDD2 } \\
\cline { 2 - 7 } & NMOS & PMOS & NMOS & PMOS & NMOS & PMOS \\
\hline 0.5 & .190 & .413 & .201 & .331 & .238 & .279 \\
\hline 1.0 & .177 & .384 & .187 & .311 & .208 & .226 \\
\hline 1.5 & .166 & .374 & .171 & .294 & .173 & .198 \\
\hline 2.0 & .161 & .372 & .159 & .280 & .146 & .184 \\
\hline 2.5 & .158 & .375 & .149 & .272 & .114 & .175 \\
\hline 3.0 & .155 & .370 & .146 & .267 & .095 & .164 \\
\hline
\end{tabular}

TABLE IV

VALUES FOR $R_{\text {ext }}(\Omega)$ VERSUS $V_{\text {gss }}$ FOR ALL THREE PROCESSES USING THE $\operatorname{SBGD}\left(I_{d s}\right)$ TECHNIQUE

\begin{tabular}{c|c|c|c|c|c|c}
\hline \hline \multirow{2}{*}{$\left|\mathrm{V}_{\text {gst }}\right|$} & \multicolumn{2}{|c|}{ CONV } & \multicolumn{2}{c|}{ LDD1 } & \multicolumn{2}{c}{ LDD2 } \\
\cline { 2 - 7 } & NMOS & PMOS & NMOS & PMOS & NMOS & PMOS \\
\hline 0.5 & 112 & 361 & 126 & 377 & 157 & 390 \\
\hline 1.0 & 94 & 294 & 108 & 314 & 136 & 320 \\
\hline 1.5 & 83 & 237 & 95 & 275 & 121 & 276 \\
\hline 2.0 & 76 & 209 & 87 & 251 & 111 & 246 \\
\hline 2.5 & 72 & 198 & 81 & 234 & 103 & 221 \\
\hline 3.0 & 68 & 190 & 78 & 218 & 98 & 204 \\
\hline
\end{tabular}

low $V_{g s t}$ drives were very similar, except that the upper $V_{g s t}$ usable was $\sim-4.0$ versus $3.5 \mathrm{~V}$ for $\mathrm{n}$-channel devices. This is due to the higher intrinsic channel resistance of the PMOS devices, so that $R_{d s}$ variations are less significant. Figs. 11 and 12 are representative plots of $d L$ and $\boldsymbol{R}_{\text {ext }}$ for the PMOS transistors. "LDD1" and "LDD2" refer to the process used to fabricate the devices, not the type of structure.

\section{Summary AND CONClusions}

This study examined the practical application of four techniques used to measure $L_{\text {eff }}$ and $R_{\text {ext }}$. Both conventional and two LDD structures fabricated in a submicrometer CMOS process were used. The results indicate that all the extraction methods are applicable to both n-channel and p-channel devices, although some are only valid over a small range of gate biases. Inconsistencies in applying $V_{t}$ calculations to the extraction equations set a lower limit for $V_{g s t}$ of $\sim 0.5 \mathrm{~V}$, while the upper limit of 2.0-4.0 V arises due to imprecision in $R_{d s}$ measurements influencing the double regression steps involved in the techniques. The SBGD $\left(I_{d s}\right)$ method was applicable over a wider range of bias conditions than the other techniques analyzed and is easier to implement, which makes it the most suitable technique. Tables III and IV give $d L$ and $R_{\text {ext }}$ data for the SBGD $\left(I_{d s}\right)$ method for all three processes and both NMOS and PMOS devices.

Unfortunately, it is difficult to independently verify the results of these measurements quantitatively. SEM techniques used on conventional device structures show good agreement with the electrical techniques [10], but are not easily applied to LDD transistors. Physical trends such as 
source/drain modulation of LDD regions indicated by the calculations qualitatively match the expected device behavior. Device simulations [6], [7] also support the accuracy of the methods. This kind of evidence from such varied sources gives support to using the methods, but does not verify their application.

\section{ACKNOWLEDGMENT}

The author wishes to express his appreciation to J. Hudak for his many helpful discussions.

\section{REFERENCES}

[1] K. Terada and H. Muta " A new method to determine effective MOSFET channel length," Japan. J. Appl. Phys., vol. 18, p. 953, 1979.

[2] J. Chern, P. Chang, R. Motta, and N. Godhino, "A new method to determine MOSFET channel length,"' IEEE Electron Device Lett., vol. EDL-1, p. 170, 1980.

[3] J. Scarpulla and J. Krusius, "Improved statistical method for extraction of MOSFET effective channel length and resistance," IEEE Trans. Electron Devices, vol. ED-34, p. 1354, 1987.

[4] B. Sheu, C. Hu, P. Ko, and F.-C. Hsu, "Source-and-drain series resistance of LDD MOSFET's," IEEE Electron Device Lett., vol. EDL-5, p. $365,1984$.

[5] G. Hu, C. Chang, and Y. Chia, "Gate-voltage dependent effective channel length and series resistance of LDD MOSFET's," IEEE Trans. Electron Devices, vol. ED-34, p. 2469, 1987.

[6] W. Wordeman, J. Sun, and S. Laux, "Geometry effects on MOSFET channel length extraction algorithms, " IEEE Electron Device Lett. vol. EDL-6, p. 186, 1985.
[7] J. Sun, M. Wordeman, and S. Laux, "On the accuracy of channel length characterization of LDD MOSFET's," IEEE Trans. Electron Devices, vol. ED-33, p. 1556, 1986

[8] S. Laux, "Accuracy of effective channel length/external resistance extraction algorithms for MOSFET's," IEEE Trans. Electron Devices, vol. ED-31, p. 1245, 1984.

[9] G. Box, W. Hunter, and J. Hunter, Statistics for Experimenters. New York: Wiley, 1978

[10] M. Karnett and R. Dunham, "An improved SEM technique for accurate determination of MOSFET channel length," Proc. SPIE, vol. 921, p. 440,1988 .

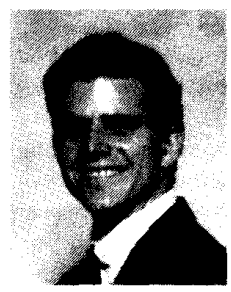

David J. Mountain was born in Davenport, IA, on September 22, 1960. He received the B.S. degree in electrical engineering from the University of Notre Dame in 1982 and the M.S. degree in electrical engineering from the University of Maryland in 1986.

He has been a Research Scientist with the Department of Defense in Fort Meade, MD, since 1982. His previous work has included investigation of silicides for VLSI technology, processing and characterization of thin gate oxides, MOS device and circuit simulation, and radiation effects in semiconductor devices and circuits. During the 1987 calendar year, he was awarded a fellowship for study at North Carolina State University in the area of solid-state electronics. His present interests include characterization of submicrometer devices and process technologies and hot-carrier effects in MOS transistors and circuits.

Mr. Mountain is a member of Eta Kappa Nu and Tau Beta Pi. 